\title{
Boundedness of log Calabi-Yau pairs of Fano type
}

\author{
Christopher D. Hacon and Chenyang Xu
}

\begin{abstract}
We prove a boundedness result for klt pairs $(X, B)$ such that $K_{X}+$ $B \equiv 0$ and $B$ is big. As a consequence we obtain a positive answer to the Effective Iitaka Fibration Conjecture for klt pairs with big boundary.
\end{abstract}

\section{Introduction}

Let $X$ be a smooth complex projective variety. The canonical ring $R\left(K_{X}\right)=$ $\oplus_{m \geq 0} H^{0}\left(\omega_{X}^{m}\right)$ is one of the fundamental birational invariants used to study the geometry of $X$. Since $R\left(K_{X}\right)$ is finitely generated ([BCHM10, CL12]), if $R\left(K_{X}\right) \neq \mathbb{C}$, then there is a natural rational map $X \rightarrow Z=\operatorname{Proj} R\left(K_{X}\right)$. This map is known as the Iitaka fibration and it is birationally equivalent to the map $\phi_{m}: X \rightarrow \mathbb{P}\left(H^{0}\left(X, m K_{X}\right)^{*}\right)$ for any $m>0$ sufficiently divisible. It is an important problem to understand for which values of $m$, the map $\phi_{m}$ is birational to the Iitaka fibration. It is classically known that for curves $m \geq 3$ suffices and for surfaces $m \geq 24$ suffices (in fact by work of Bombieri, if $X$ is a surface of general type, then $m \geq 5$ is sufficient). This leads to the following natural conjecture (cf. [HM06]).

Conjecture 1.1. Fix a positive integer $n$. There exists an integer $k>0$, which only depends on $n$, such that if $X$ is an n-dimensional smooth complex projective variety, then $\left|k K_{X}\right|$ defines a map birational to the Iitaka fibration.

For threefolds, the conjecture is answered affirmatively in [Kaw86, FM00, VZ09, CC10]. We remark that for threefolds, such $m$ can be computed explicitly. However, in many cases finding the optimal bound is a difficult task. Furthermore, in [Tsu06, HM06, Tak06], Conjecture 1.1 is verified for varieties of general type and arbitrary dimension, however no explicit expression for $m$ is known. Finally, the recent work [BZ14] gives a positive answer under the additional assumption that the general fiber $F$ of the Iitaka fibration is bounded in an appropriate sense (more precisely, the smallest integer $b>0$ 
such that $\left|b K_{F}\right| \neq 0$ is bounded as well as the middle Betti number of a desingularization of the corresponding degree $b$ cyclic cover $\tilde{F} \rightarrow F$ ).

In many applications, it is important to study log pairs. If we consider the pairs $(X, B)=\left(\mathbb{P}^{1}, P_{1}+P_{2}+t P_{3}\right)$ where the $P_{i}$ are distinct points on $\mathbb{P}^{1}$ and $t \in \mathbb{Q}_{>0}$, then $\left|m\left(K_{X}+B\right)\right|$ is birational only for $m \geq \frac{1}{t}$. Therefore, the analog of (1.1) for log canonical pairs does not hold unless we put some restriction on the coefficients. As observed in [Kol94], the right condition for the coefficients of the boundary should be that they satisfy the descending chain condition (DCC) i.e., every non inecreasing sequence is eventually constant. With this assumption, we can extend the above conjecture to the context of log pairs.

Conjecture 1.2 (Effective Log Iitaka Fibration Conjecture). Fix a positive integer $n \in \mathbb{N}$ and a DCC set $I \subset[0,1] \cap \mathbb{Q}$. There exists an integer $m>0$ which only depends on $n$ and $I$ such that if $(X, B)$ is an n-dimensional complex projective log canonical pair such that the coefficients of $B$ are in $I$ and $\kappa\left(K_{X}+B\right) \geq 0$, then $\left|m\left(K_{X}+B\right)\right|$ defines a map birational to the Iitaka fibration.

Various low dimensional cases of Conjecture 1.2 are known (see [Kol94, Ale94, Jia13, Tod10, TX09]). In arbitrary dimension, we also have an affirmative answer in the log general type case (cf. [HMX13, HMX14]). However, the case of intermediate Kodaira dimension is more complicated and very little is known in arbitrary dimension. The well-known strategy, first developed in [FM00], is to use the canonical bundle formula, which is a far reaching generalization of Kodaira's formula for elliptic fibrations, to reduce the above question to questions about the general fiber and the base pair which is of log general type.

More precisely, let $f: X \rightarrow Z$ be a morphism birational to the Iitaka fibration, then the pluricanonical ring $R\left(K_{X}+B\right)$ can be described in terms of the pluricanonical type ring $R\left(K_{Z}+B_{Z}+M_{Z}\right)$ where $\left(Z, B_{Z}\right)$ is a klt pair, the coefficients of $B_{Z}$ belong to an appropriate DCC set and $K_{Z}+$ $B_{Z}+M_{Z}$ is big. Here $B_{Z}$ denotes the boundary part which measures the singularities of the map $f$ and $M_{Z}$ is the moduli part which measures the variation of the fibers of $f:(X, B) \rightarrow Z$. If one can show that $\left(Z, B_{Z}+M_{Z}\right)$ is also a log canonical pair and the coefficients of $M_{Z}$ also belong to a DCC (or finite) set, then the result would follow from the results in [HMX13, HMX14]. Therefore, the main difficulty of this approach is to control the corresponding moduli divisor $M_{Z}$. This is a difficult question. An affirmative answer would follow from the effective adjunction conjectures in [PS06, Conjecture 7.13]. 
In [BZ14], the authors treat $M_{Z}$ as a divisor class (rather than an actual divisor) and develop results similar to those of [HMX14] in this context.

In this note, we show that the results of [HMX14] imply a (strong) boundedness result for klt pairs $(X, B)$ such that $K_{X}+B \equiv 0$ where $B$ is big. Before stating the main result we fix some notation. Given a DCC set $I \subset[0,1] \cap \mathbb{Q}$ and a positive integer $n \in \mathbb{N}$, let $\mathcal{D}(n, I)$ be the set of all $n$ dimensional projective klt pairs $(X, B)$ such that $B$ is big, the coefficients of $B$ are contained in $I$ and $\kappa\left(K_{X}+B\right)=0$. Let $\mathcal{C}(n, I)$ be the subset of $\mathcal{D}(n, I)$ consisting of those pairs $(X, B)$ satisfying $K_{X}+B \equiv 0$.

Theorem 1.3. Fix $I \subset[0,1] \cap \mathbb{Q}$ a DCC set and $n \in \mathbb{N}$. There exists an integer $k>0$ which only depends on $I$ and $n$ such that if $(X, B) \in \mathcal{D}(n, I)$, then $h^{0}\left(k\left(K_{X}+B\right)\right)=1$. Furthermore, the set $\mathcal{C}(n, I)$ is bounded (cf. Section 2.2).

As a consequence, by [BZ14], we also obtain a positive answer for (1.2) when the general fibers of the Iitaka fibration have a big boundary.

Theorem 1.4. Fix a DCC set $I \subset[0,1] \cap \mathbb{Q}$ and an integer $n \in \mathbb{N}$. Then there is an integer $k>0$ depending only on $n$ and $I$ such that if

1) $(X, B)$ is an $n$-dimensional projective klt pair,

2) the coefficients of $B$ are in $I$,

3) $\kappa\left(K_{X}+B\right) \geq 0$ and $(X, B) \rightarrow Z$ is the Iitaka fibration, and

4) if $\eta$ is the generic point of $Z$ and $X_{\eta}$ the corresponding fiber, then $B_{\eta}:=\left.B\right|_{X_{\eta}}$ is big (see Definition 2.3),

then $\left|k\left(K_{X}+B\right)\right|$ is birational to the Iitaka fibration of $(X, B)$.

As an immediate corollary of (1.3), we obtain the following result which can be used to generalize [BL15, Theorem 5.2] from birational boundedness to boundedness (see [BL15, 3.4]).

Corollary 1.5. Fix two positive integers $n$ and $m$. Consider the set of $n$ dimensional weak Fano varieties $X$ with Cartier index $m$, i.e., $X$ is klt, $-K_{X}$ is big and nef and $-m K_{X}$ is Cartier. Then the set of all such $X$ is bounded. 


\section{Acknowledgements}

We would like to thank Lev Borisov and James $\mathrm{M}^{\mathrm{c}}$ Kernan for helpful communications. We also want to thank the referees for several useful suggestions. The first author was partially supported by NSF research grants no: DMS-1300750, DMS-1265285, FRG grant no: DMS-1265285 and a grant from the Simons foundation, the second author was partially supported by the grant 'The Recruitment Program of Global Experts' and 'The Chinese National Science Fund for Distinguished Young Scholars'. A large part of this work was done when the second author visited IAS, which was partially sponsored by Ky Fan and Yu-Fen Fan Membership Funds, S.S. Chern Fundation and NSF: DMS-1128155, 1252158.

\section{Preliminaries}

\subsection{Notation and Conventions}

Notation 2.1. We follow the usual notation from [KM98] and [BCHM10]. We say that a pair $(X, B)$ is $\epsilon$-klt ( $\epsilon$ Kawamata log terminal), if its total discrepancy is larger than $-1+\epsilon$.

Let $(X, B)$ be a log pair and $B_{i}$ be the components of the support of $B$. We say that $(X, B)$ is log smooth if $X$ is smooth and $B$ has snc (simple normal crossing) support. Given a $\log$ smooth pair $(X, B)$, we say that $V \subset X$ is a stratum of $(X, B)$ if either $V=X$ or $V$ is a component of $B_{i_{1}} \cap \ldots \cap B_{i_{r}}$. We say that a $\log$ smooth pair $(Y, D)$ with a morphism $f: Y \rightarrow S$ is $\log$ smooth over a smooth variety $S$ if every stratum of $(Y, D)$ (including $Y$ ) is smooth over $S$.

Let $X$ and $X^{\prime}$ be two normal projective varieties. We say that a birational map $f: X \rightarrow X^{\prime}$ is small if both $f$ and $f^{-1}$ do not contract any divisor, i.e., $X$ and $X^{\prime}$ are isomorphic in codimension 1.

If $X \rightarrow X^{\prime}$ is a small birational map between normal $\mathbb{Q}$-factorial projective varieties, then we say that $X^{\prime}$ is an $S Q M$ (small $\mathbb{Q}$-factorial modification) of $X$. If $X^{\prime} \rightarrow X$ is a small birational morphism of normal quasiprojective varieties such that $X^{\prime}$ is $\mathbb{Q}$-factorial, then we say that $X^{\prime}$ is a $\mathbb{Q}$-factorialization of $X$.

If $f: X \rightarrow S$ is a morphism and $s \in S$ is a point (not necessarily closed), then we denote $X_{s}=X \times_{S} s$. If $L$ is a line bundle, then we denote $L_{s}=\left.L\right|_{X_{s}}$. If $f$ is equidimensional with reduced fibers, $s \in S$ is a smooth point, $B$ is a $\mathbb{Q}$-divisor whose support does not contain any component of $X_{s}$, then we denote by $B_{s} \subset X_{s}$ the divisor on $X_{s}$ given by $B \cdot X_{s}$. 
Definition 2.2. Let $X$ be a normal projective variety, $L$ be a $\mathbb{Q}$-Cartier divisor. If we assume $\kappa(L) \geq 0$, then there is a rational map $\phi: X \rightarrow Z$ such that $\operatorname{dim} Z=\kappa(L)$ and $\kappa\left(\left.L\right|_{X_{z}}\right)=0$ where $z$ is a very general point of $Z$. This map is defined (up to birational isomorphism) by $|k L|$ for $k>0$ sufficiently divisible. We say that $\phi$ is the Iitaka fbration of $L$ (see [Laz04, $2.1 \mathrm{C}])$.

Definition 2.3. For an effective $\mathbb{Q}$-divisor $B$ on $X$ and a proper rational map $X \rightarrow Z$, we say that $B$ is big over the generic point $\eta$ of $Z$, if for some resolution $g: X^{\prime} \rightarrow X$ inducing a morphism $X^{\prime} \rightarrow Z$, the restriction of the divisor $B^{\prime}=g_{*}^{-1} B+\operatorname{Ex}(g)$ to the generic fiber $X_{\eta}^{\prime}$ is big. Alternatively, this is equivalent to saying that for such $X^{\prime}$ there is a relatively big divisor $B^{\prime}$ over $Z$ satisfying $g_{*} B^{\prime}=B$.

\subsection{Boundedness}

We say that a collection of $\log$ pairs $\mathcal{C}$ is bounded if there exists a pair $(\mathcal{X}, \mathcal{B})$ and a projective morphism of quasi-projective schemes $f: \mathcal{X} \rightarrow S$ such that any $(X, B) \in \mathcal{C}$ is isomorphic to the fiber $\left(\mathcal{X}_{s}, \mathcal{B}_{s}\right)$ of $f$ for some closed point $s \in S$.

Proposition 2.4. Fix $\epsilon>0$. Let $f: \mathcal{X} \rightarrow S$ be a morphism of quasiprojective varieties, $\mathcal{B}$ a divisor on $\mathcal{X}$. Assume that there is a dense set of points $s_{i} \in S(i \in I)$ such that each $\left(\mathcal{X}_{s_{i}}, \mathcal{B}_{s_{i}}\right)$ is an $\epsilon$-klt pair. Then there exists a dense open set $U \subset S$ such that $\left.\left(K_{\mathcal{X}}+\mathcal{B}\right)\right|_{\mathcal{X} \times{ }_{S} U}$ is $\mathbb{Q}$-Cartier and klt.

Proof. After replacing $S$ by an open subset, we can assume that $(\mathcal{X}, \mathcal{B})$ has a $\log$ resolution $\mu: \mathcal{Y} \rightarrow \mathcal{X}$ such that $\left(\mathcal{Y}, \mu_{*}^{-1} \mathcal{B}+\mathcal{E}\right)$ is $\log$ smooth over $S$, where $\mathcal{E}$ is the sum of the $\mu$-exceptional divisors. We run a minimal model program for $K_{\mathcal{Y}}+\mu_{*}^{-1} \mathcal{B}+(1-\epsilon) \mathcal{E}$ over $\mathcal{X}$ which, by [BCHM10], terminates with a relative minimal model $g: \mathcal{X}^{\prime} \rightarrow \mathcal{X}$.

Let $\nu: \mathcal{Y} \rightarrow \mathcal{X}^{\prime}$ be the induced birational map. Since $K_{\mathcal{X}^{\prime}}+\nu_{*}\left(\mu_{*}^{-1} \mathcal{B}+\right.$ $(1-\epsilon) \mathcal{E})$ is nef over $\mathcal{X}$, it follows that

$$
\left.\left(K_{\mathcal{X}^{\prime}}+\nu_{*}\left(\mu_{*}^{-1} \mathcal{B}+(1-\epsilon) \mathcal{E}\right)\right)\right|_{\mathcal{X}_{s_{i}}^{\prime}}
$$


is nef over $\mathcal{X}_{s_{i}}$. Let $g_{i}: \mathcal{X}_{s_{i}}^{\prime} \rightarrow \mathcal{X}_{s_{i}}$ be the induced morphism.

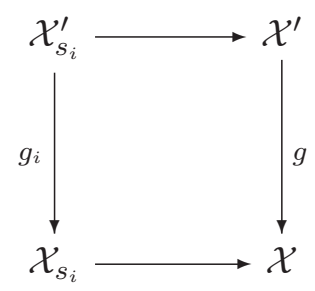

Since $\nu_{*} \mathcal{E}$ is $g$ exceptional, by semicontinuity of the fiber dimension, it follows that after restricting over an appropriate open subset of $S,\left.\nu_{*} \mathcal{E}\right|_{\mathcal{X}_{s_{i}}^{\prime}}$ is $g_{i}$ exceptional. Since

$$
\left.\left(K_{\mathcal{X}^{\prime}}+\nu_{*}\left(\mu_{*}^{-1} \mathcal{B}+(1-\epsilon) \mathcal{E}\right)\right)\right|_{\mathcal{X}_{s_{i}}^{\prime}}-g_{i}^{*}\left(K_{\mathcal{X}_{s_{i}}}+B_{s_{i}}\right)
$$

is an effective divisor whose support equals the exceptional divisor $\left.\nu_{*} \mathcal{E}\right|_{\mathcal{X}_{s_{i}}^{\prime}}$, by the negativity lemma, it follows that $\left.\nu_{*} \mathcal{E}\right|_{\mathcal{X}_{s_{i}}^{\prime}}=0$ and $\mathcal{X}_{s_{i}}^{\prime} \rightarrow \mathcal{X}_{s_{i}}$ is a small birational $\left(K_{\mathcal{X}_{s_{i}}^{\prime}}+\left.\left(\nu_{*} \mu_{*}^{-1} \mathcal{B}\right)\right|_{\mathcal{X}_{s_{i}}^{\prime}}\right)$-trivial morphism.

Let $\phi: \overline{\mathcal{X}} \rightarrow \mathcal{X}$ be the relative $\log$ canonical model of $K_{\mathcal{X}^{\prime}}+\nu_{*} \mu_{*}^{-1} \mathcal{B}$ over $\mathcal{X}$. Since $\phi$ contracts all $\left(K_{\mathcal{X}^{\prime}}+\nu_{*} \mu_{*}^{-1} \mathcal{B}\right)$-trivial curves over $\mathcal{X}$, it follows that $\overline{\mathcal{X}}_{s_{i}}=\mathcal{X}_{s_{i}}$. Therefore, after possibly shrinking $S$, we may assume that $\nu_{*} \mathcal{E}=0$ and $\phi: \overline{\mathcal{X}} \rightarrow \mathcal{X}$ is an isomorphism. In particular $K_{\mathcal{X}}+\mathcal{B}=$ $g_{*}\left(K_{\mathcal{X}^{\prime}}+\nu_{*} \mu_{*}^{-1} \mathcal{B}\right)$ is $\mathbb{Q}$-Cartier. Finally, we may assume that $K_{\mathcal{X}}+\mathcal{B}$ is klt as it is the relative $\log$ canonical model of the klt pair $K \mathcal{Y}+\mu_{*}^{-1} \mathcal{B}+(1-$ $\epsilon) \mathcal{E}$.

Proposition 2.5. Fix $\epsilon>0$. Let $f: \mathcal{X} \rightarrow S$ be a projective morphism of quasi-projective varieties and $\mathcal{B}$ a divisor on $\mathcal{X}$. Assume that there is a set of points $s_{i} \in S(i \in I)$ such that each $\left(\mathcal{X}_{s_{i}}, \mathcal{B}_{s_{i}}\right)$ is an $\epsilon$-klt pair. Then there exists a family $(\mathcal{Y}, \mathcal{D}) \rightarrow T$ over a quasi-projective base $T$ such that for any given proper birational morphism $Y \rightarrow \mathcal{X}_{s_{i}}$ whose exceptional set consists of finitely many divisors with non-positive discrepancies with respect to $\left(\mathcal{X}_{s_{i}}, \mathcal{B}_{s_{i}}\right)$, there exists a closed point $t \in T$ and a birational morphism $\mu_{t}$ : $\mathcal{Y}_{t} \rightarrow \mathcal{X}_{s_{i}}$ such that the birational map $\nu_{t}: \mathcal{Y}_{t} \rightarrow Y$ is small and $K \mathcal{Y}_{t}+\mathcal{D}_{t}=$ $\mu_{t}^{*}\left(K_{\mathcal{X}_{s_{i}}}+\mathcal{B}_{s_{i}}\right)$.

Proof. Replacing $S$ by a component of the closure of $\left\{s_{i}\right\}_{i \in I}$, we may assume that $\left\{s_{i}\right\}_{i \in I} \subset S$ is dense. By Noetherian induction it suffices to prove the claim over any dense open subset of $S$. By Proposition 2.4, we may assume that $K_{\mathcal{X}}+\mathcal{B}$ is $\mathbb{Q}$-Cartier and klt. Let $\nu: \mathcal{X}^{\prime} \rightarrow \mathcal{X}$ be a $\log$ resolution and 
write

$$
K_{\mathcal{X}^{\prime}}+\mathcal{D}^{\prime}=\nu^{*}\left(K_{\mathcal{X}}+\mathcal{B}\right)+\mathcal{E}
$$

where $\mathcal{D}^{\prime}$ and $\mathcal{E}$ are effective with no common components. After a (possibly non-surjective) dominant base change, we may assume that $\left(\mathcal{X}^{\prime}, \mathcal{D}^{\prime}\right) \rightarrow S$ is $\log$ smooth with geometrically irreducible strata over $S$, and the induced morphism from the corresponding fiber $\nu_{i}: \mathcal{X}_{s_{i}}^{\prime} \rightarrow \mathcal{X}_{s_{i}}$ is a log resolution of $\left(\mathcal{X}_{s_{i}}, \mathcal{B}_{s_{i}}\right)$ (see e.g. [HMX13], bottom of page 1105). Furthermore, after a possible further blow up, we can assume $\left(\mathcal{X}^{\prime}, \mathcal{D}^{\prime}\right)$ is terminal (see e.g., the beginning of the proof of Theorem 1.8 in [HMX13]).

Note that by our assumption on the existence of the simultaneous log resolution $\mathcal{Y}$, the divisors of discrepancy $a\left(E, \mathcal{X}_{s_{i}}, \mathcal{B}_{s_{i}}\right) \leq 0$ are in one to one correspondence with divisors $\mathcal{E}$ over $\mathcal{X}$ such that $a(\mathcal{E}, \mathcal{X}, \mathcal{B}) \leq 0$ and all of these divisors are divisors on $\mathcal{X}^{\prime}$. Denote this set of divisors by $\mathcal{F}$. By [BCHM10, 1.4.3], for any fixed subset $\mathcal{F}^{\prime} \subset \mathcal{F}$ there exists a birational map $\mathcal{X}^{\prime} \rightarrow \mathcal{X}_{\mathcal{F}^{\prime}}$ over $\mathcal{X}$ such that $\mathcal{X}_{\mathcal{F}^{\prime}} \rightarrow \mathcal{X}$ contracts precisely the divisors in $\mathcal{F}^{\prime}$. There exists a dense open set $U \subset S$ such that for any $t \in U$ and any $\mathcal{F}^{\prime} \subset \mathcal{F},\left(\mathcal{X}_{\mathcal{F}^{\prime}}\right)_{t} \rightarrow \mathcal{X}_{t}$ contracts precisely the divisors $\left(\mathcal{E}_{i}\right)_{t}$ for $\mathcal{E}_{i} \in \mathcal{F}^{\prime}$. Letting $\mathcal{D}_{\mathcal{F}^{\prime}}^{\prime}$, be the pushforward of $\mathcal{D}^{\prime}$ on $\mathcal{X}_{\mathcal{F}^{\prime}}$, defining

$$
T=\sqcup_{\mathcal{F}^{\prime} \subset \mathcal{F}} U_{\mathcal{F}^{\prime}} \quad \text { where } \quad U_{\mathcal{F}^{\prime}}=U
$$

and

$$
\left.(\mathcal{Y}, \mathcal{D})\right|_{U_{\mathcal{F}^{\prime}}}=\left(\mathcal{X}_{\mathcal{F}^{\prime}}, \mathcal{D}_{\mathcal{F}^{\prime}}^{\prime}\right) \times_{S} U
$$

and continuing by Noetherian induction, we obtain the required family $(\mathcal{Y}, \mathcal{D}) \rightarrow T$.

\subsection{Families of varieties of Fano type}

Definition 2.6. A projective normal variety $X$ is of Fano type, if there exists an effective big $\mathbb{Q}$-divisor $B$ such that $K_{X}+B \sim_{\mathbb{Q}} 0$ and $(X, B)$ is klt.

If $X$ is of Fano type, then as $B$ is big, for any ample divisor $A$, there exists a sufficiently small rational number $\epsilon>0$, such that $B-\epsilon A \sim_{\mathbb{Q}} G \geq 0$. For $0<\delta \ll 1$, let $B^{\prime}=(1-\delta) B+\delta G$. It follows easily that $\left(X, B^{\prime}\right)$ is klt and since

$$
-\left(K_{X}+B^{\prime}\right) \sim_{\mathbb{Q}} \delta \epsilon A
$$

the pair $\left(X, B^{\prime}\right)$ is a log Fano variety. In particular, if $X$ is also $\mathbb{Q}$-factorial, then by [BCHM10, 1.3.2], $X$ is a Mori Dream space. Thus the number of 
SQM (small $\mathbb{Q}$-factorial modifications) of $X$ is finite. Moreover, the Mori chamber decomposition of $\operatorname{Mov}(X)$ is polyhedral, such that each SQM corresponds to a maximal dimensional chamber of $\operatorname{Mov}(X)$ (see $[\mathrm{HK} 00,1.10]$ ).

Lemma 2.7. Let $f: \mathcal{X} \rightarrow S$ be a projective morphism over an affine variety $S$, such that the geometric generic fiber is of Fano type. Then there is a dense open subset $S^{0} \subset S$ such that for any line bundle $L$ on $\mathcal{X}$ and for sufficiently divisible $m$, the map

$$
H^{0}\left(\mathcal{X}, L^{\otimes m}\right) \rightarrow H^{0}\left(\mathcal{X}_{s}, L_{s}^{\otimes m}\right)
$$

is surjective for any $s \in S^{0}$.

Proof. Let $\eta \in S$ be the generic point, $K$ the algebraic closure of $k(\eta)$ and $\mathcal{X}_{K}=\mathcal{X} \times{ }_{S} \operatorname{Spec}(K)$. By assumption there is an effective big $\mathbb{Q}$-divisor $\mathcal{D}_{K}$ on $\mathcal{X}_{K}$ such that $K_{\mathcal{X}_{K}}+\mathcal{D}_{K} \sim_{\mathbb{Q}} 0$ and $\left(\mathcal{X}_{K}, \mathcal{D}_{K}\right)$ is klt. Therefore, we may find a finite field extension $k(\eta) \subset K^{\prime} \subset K$ such that $\mathcal{D}_{K}$ is induced by base extension from $K^{\prime}$, i.e.,

$$
\mathcal{D}_{K}=\mathcal{D}_{K^{\prime}} \times_{\operatorname{Spec}\left(K^{\prime}\right)} \operatorname{Spec}(K),
$$

where $\mathcal{D}_{K^{\prime}}$ is defined over $K^{\prime}$. Thus if we denote by $X_{K^{\prime}}=X_{\eta} \times{ }_{\eta} \operatorname{Spec}\left(K^{\prime}\right)$, then $K_{\mathcal{X}_{K^{\prime}}}+\mathcal{D}_{K^{\prime}} \sim_{\mathbb{Q}} 0$ and $\left(\mathcal{X}_{K^{\prime}}, \mathcal{D}_{K^{\prime}}\right)$ is klt.

By the flat base change theorem, it is clear that to prove Lemma 2.7, it suffices to prove that there exists a dominant quasi-finite base change $T \rightarrow S$ and

$$
H^{0}\left(\mathcal{X} \times{ }_{S} T, L^{\otimes m}\right) \rightarrow H^{0}\left(\mathcal{X}_{t}, L_{t}^{\otimes m}\right)
$$

is surjective for any $t \in T^{0}$, where $T^{0}$ is a dense open set of $T$. Therefore, after replacing $S$ by a quasi-finite dominant base change, we can assume that $S$ is affine and there is a big boundary $\mathcal{D}$, such that $K_{\mathcal{X}}+\mathcal{D} \sim_{\mathbb{Q}, S} 0$ and $(\mathcal{X}, \mathcal{D})$ is klt. We may further assume that $\mathcal{D} \sim_{\mathbb{Q}} \mathcal{A}+\mathcal{B}$ where $\mathcal{A}$ is ample, $(\mathcal{X}, \mathcal{B})$ is klt and that $\left(\mathcal{X}_{s}, \mathcal{B}_{s}\right)$ is klt for any $s \in S$ after a possibly further shrinking of $S$. We take a $\log$ resolution $\psi: \mathcal{X}^{\prime} \rightarrow \mathcal{X}$ of $(\mathcal{X}, \mathcal{B})$ and write

$$
\psi^{*}\left(K_{\mathcal{X}}+\mathcal{B}\right)+\mathcal{E}=K_{\mathcal{X}^{\prime}}+\mathcal{D}^{\prime}
$$

where $\mathcal{E}, \mathcal{D}^{\prime}$ are effective and do not have common components. We can assume that $\left(\mathcal{X}^{\prime}, \mathcal{D}^{\prime}\right)$ is terminal. By shrinking $S$, we may assume that $\left(\mathcal{X}^{\prime}, \mathcal{D}^{\prime}\right)$ is $\log$ smooth over $S$ and for any $s \in S, \mathcal{X}_{s}^{\prime} \rightarrow \mathcal{X}_{s}$ is a $\log$ resolution of $\left(\mathcal{X}_{s}, \mathcal{B}_{s}\right)$. 
Let $L$ be a line bundle on $\mathcal{X}$, then for any rational number $0<\lambda \ll 1$, the $\mathbb{Q}$-divisor $\mathcal{A}_{\lambda}$ : $=\mathcal{A}+\lambda L$ is ample. By [HMX13, 1.8], for sufficiently divisible $m$, the morphism

$$
\begin{aligned}
& H^{0}\left(\mathcal{X}^{\prime}, \mathcal{O}_{\mathcal{X}^{\prime}}\left(m\left(K_{\mathcal{X}^{\prime}}+\mathcal{D}^{\prime}+\psi^{*} \mathcal{A}_{\lambda}\right)\right)\right) \\
\rightarrow & H^{0}\left(\mathcal{X}_{s}^{\prime}, \mathcal{O}_{\mathcal{X}_{s}^{\prime}}\left(\left.m\left(K_{\mathcal{X}^{\prime}}+\mathcal{D}^{\prime}+\psi^{*} \mathcal{A}_{\lambda}\right)\right|_{\mathcal{X}_{s}^{\prime}}\right)\right)
\end{aligned}
$$

is surjective. Since

$$
\psi_{*}\left(K_{\mathcal{X}^{\prime}}+\mathcal{D}^{\prime}+\psi^{*} \mathcal{A}_{\lambda}\right)=K_{\mathcal{X}}+\mathcal{B}+\mathcal{A}_{\lambda} \sim_{\mathbb{Q}, S} \lambda L
$$

and

$$
K_{\mathcal{X}^{\prime}}+\mathcal{D}^{\prime}+\psi^{*} \mathcal{A}_{\lambda}-\psi^{*}\left(K_{\mathcal{X}}+\mathcal{B}+\mathcal{A}_{\lambda}\right)
$$

is effective and $\psi$-exceptional, this implies that

$$
H^{0}\left(\mathcal{X}, L^{\otimes m}\right) \rightarrow H^{0}\left(\mathcal{X}_{s}, L_{s}^{\otimes m}\right)
$$

is surjective for any sufficiently divisible integer $m>0$.

Proposition 2.8. Let $f: \mathcal{X} \rightarrow S$ be a projective morphism of quasiprojective varieties such that the geometric generic fiber is of Fano type. Assume that there is a dense set of points $\left\{s_{i}\right\}_{i \in I} \subset S$ such that each fiber $\mathcal{X}_{s_{i}}$ is $\mathbb{Q}$-factorial.

Then there exists a dominant morphism $T \rightarrow S$, such that if we denote $\mathcal{X}_{T}=\mathcal{X} \times{ }_{S} T$, then for any $t \in T$,

1) the morphism $\rho_{t}: N^{1}\left(\mathcal{X}_{T} / T\right) \rightarrow N^{1}\left(\mathcal{X}_{t}\right)$ is an isomorphism,

2) $\rho_{t}\left(\operatorname{Mov}\left(\mathcal{X}_{T} / T\right)\right)=\operatorname{Mov}\left(\mathcal{X}_{t}\right)$ and there is a one-to-one correspondence between the two Mori chamber decompositions.

Proof. By the proof of (2.7), after a dominant base change, we may assume that $S$ is affine, there is a big boundary $\mathcal{B}$ over $S$ such that $(\mathcal{X}, \mathcal{B})$ is a klt pair, projective over $S$, each fiber $\left(\mathcal{X}_{s}, \mathcal{B}_{s}\right)$ is a klt pair and

$$
K_{\mathcal{X}}+\mathcal{B} \sim_{\mathbb{Q}, S} 0
$$

After replacing $S$ by a dense open set, it follows from [KM92, 12.1.7] and our assumptions that every fiber $\mathcal{X}_{s}$ is $\mathbb{Q}$-factorial. 
By $[\mathrm{dFH} 11,6.6]$, after restricting over a dense open set, we can assume that for any $s \in S$, the morphisms

$$
N^{1}(\mathcal{X} / S) \rightarrow N^{1}\left(\mathcal{X}_{s}\right) \text { and } N_{1}(\mathcal{X} / S) \rightarrow N_{1}\left(\mathcal{X}_{s}\right)
$$

are isomorphisms. We note that the general setting in [dFH11] assumes that the fibers are terminal $\mathbb{Q}$-factorial Fano varieties and the base $S$ is a curve. However, the argument of $[\mathrm{dFH} 11,6.6]$ only needs $H^{1}\left(\mathcal{X}_{s}, \mathcal{O}_{\mathcal{X}_{s}}\right)=0$ and $\mathcal{X}_{s}$ being $\mathbb{Q}$-factorial, and it can be indeed applied to a base of any dimension. This gives (1).

By Lemma 2.7, we can assume that (after a possible base change),

$$
\rho_{s}: \overline{\operatorname{Eff}}(\mathcal{X} / S) \rightarrow \overline{\operatorname{Eff}}\left(\mathcal{X}_{s}\right)
$$

is an isomorphism for every $s \in S$ (here $\overline{\operatorname{Eff}}(\mathcal{X} / S)$ denotes the relative pseudoeffective cone i.e. the closure of the relative big cone). Let $\mathcal{X}^{1}, \mathcal{X}^{2}, \ldots, \mathcal{X}^{k}$ be the $\mathbb{Q}$-factorial birational models corresponding to the maximal dimensional chambers of the Mori chamber decomposition of $\operatorname{Mov}(\mathcal{X} / S)$. Each $\mathcal{X}^{i}$ is also of Fano type, in particular, it is a Mori dream space (see [BCHM10, 1.3.2]). The nef cones $\operatorname{Nef}\left(\mathcal{X}^{i} / S\right)(1 \leq i \leq k)$ are finitely generated polyhedral cones and give the Mori chamber decomposition of $\operatorname{Mov}(\mathcal{X} / S)$.

By shrinking $S$, we assume that for any $s \in S$, any $1 \leq i \leq k, \mathcal{X}_{s} \rightarrow \mathcal{X}_{s}^{i}$ is a small birational map, which implies

$$
\rho_{s}(\operatorname{Mov}(\mathcal{X} / S)) \subset \operatorname{Mov}\left(\mathcal{X}_{s}\right) .
$$

Furthermore, by Lemma 2.7, we can assume that for any line bundle $L$ on $\mathcal{X}$, for any sufficiently divisible integer $d>0$

$$
H^{0}\left(\mathcal{X}, L^{\otimes d}\right) \rightarrow H^{0}\left(\mathcal{X}_{s}, L_{s}^{\otimes d}\right),
$$

is surjective for any $s \in S$.

If there is a movable divisor class $L_{s}$ on $\mathcal{X}_{s}$, then by our assumption it is the restriction of an effective divisor $L$ on $\mathcal{X}$. If $L$ is not movable, then the stable base locus $\mathbf{B}(\mathcal{X} / S, L)$ contains a divisor, which implies that $\mathbf{B}\left(\mathcal{X}_{s}, L_{s}\right)$ contains a divisor (since we have assumed that $H^{0}\left(\mathcal{X}, L^{\otimes d}\right) \rightarrow H^{0}\left(\mathcal{X}_{s}, L_{s}^{\otimes d}\right)$ is surjective for any $s \in S$ ). But this contradicts the assumption that $L_{s}$ is movable. Therefore, $L$ is movable on $\mathcal{X}$ and so $\rho_{s}(\operatorname{Mov}(\mathcal{X} / S))=\operatorname{Mov}\left(\mathcal{X}_{s}\right)$.

Replacing $S$ by an open subset, we may assume that for any $s \in S$ and $1 \leq i \leq k$, the $\mathcal{X}_{s}^{i}$ are mutually not isomorphic. Thus the cones $\operatorname{Nef}\left(\mathcal{X}_{s}^{i}\right)$ give different Mori chambers in $\operatorname{Mov}\left(\mathcal{X}_{s}\right)$. This completes the proof of $(2)$. 
Proposition 2.9. Let $(\mathcal{X}, \mathcal{B})$ be a log pair and $f: \mathcal{X} \rightarrow S$ be a projective morphism of quasi-projective varieties. Let $s_{i} \in S(i \in I)$ be a collection of points such that $\left(\mathcal{X}_{s_{i}}, \mathcal{B}_{s_{i}}\right)$ is a $\mathbb{Q}$-factorial $\epsilon$-klt pair, $K_{\mathcal{X}_{s_{i}}}+\mathcal{B}_{s_{i}} \sim_{\mathbb{Q}} 0$ and $\mathcal{B}_{s_{i}}$ is big. Then there exists a $\log$ pair $(\mathcal{Y}, \mathcal{D})$ and $g: \mathcal{Y} \rightarrow T$ a projective morphism of quasi-projective schemes such that if $\eta: \mathcal{X}_{s_{i}} \rightarrow Y$ is a small birational map and $K_{Y}+\eta_{*} \mathcal{B}_{s_{i}}$ is $\mathbb{Q}$-Cartier, then there is a point $t \in T$ such that $Y=\mathcal{Y}_{t}$ and $\eta_{*} \mathcal{B}_{s_{i}}=\mathcal{D}_{t}$.

Proof. By Noetherian induction, we can replace $S$ by a component of the closure of $\left\{s_{i}\right\}_{i \in I} \subset S$ and assume that $\left\{s_{i}\right\}_{i \in I} \subset S$ is dense. Furthermore, by Noetherian induction, it suffices to prove the assertion over some non-empty open subset of $S$. In particular, we may assume that $S$ is affine.

We first only consider small $\mathbb{Q}$-factorial modifications. By Proposition 2.4, we can assume that $(\mathcal{X}, \mathcal{B})$ is klt. Thus we can fix $a>0$ such that $K_{\mathcal{X}}+$ $(1+a) \mathcal{B}$ is klt. Let $\nu: \mathcal{X}^{\prime} \rightarrow \mathcal{X}$ be a $\log$ resolution of $(\mathcal{X}, \mathcal{B})$ and write

$$
K_{\mathcal{X}^{\prime}}+\mathcal{D}^{\prime}=\nu^{*}\left(K_{\mathcal{X}}+(1+a) \mathcal{B}\right)+\mathcal{E}
$$

where $\mathcal{D}^{\prime}$ and $\mathcal{E}$ are effective with no common components. As in the proof of Proposition 2.5, we may assume that $\left(\mathcal{X}^{\prime}, \mathcal{D}^{\prime}\right)$ is $\log$ smooth over $S$. By [HMX13, 1.8], if $m \mathcal{D}^{\prime}$ is integral, then

$$
h^{0}\left(\mathcal{O}_{\mathcal{X}_{s}}\left(\left.m\left(K_{\mathcal{X}}+(1+a) \mathcal{B}\right)\right|_{\mathcal{X}_{s}}\right)\right)=h^{0}\left(\mathcal{O}_{\mathcal{X}_{s}^{\prime}}\left(\left.m\left(K_{\mathcal{X}^{\prime}}+\mathcal{D}^{\prime}\right)\right|_{\mathcal{X}_{s}^{\prime}}\right)\right)
$$

is locally constant for $s \in S$. Therefore $K_{\mathcal{X}_{\eta}}+(1+a) \mathcal{B}_{\eta}$ is big where $\eta$ is the generic point of $S$. By Lemma 2.7, we also see that

$$
K_{\mathcal{X}_{\eta}}+\mathcal{B}_{\eta} \sim_{\mathbb{Q}} 0
$$

Therefore $\mathcal{B}_{\eta}$ is big. By shrinking $S$, we can assume that $K_{\mathcal{X}}+\mathcal{B} \sim_{\mathbb{Q}, S} 0$.

Since the SQMs of a Mori dream space are determined by the maximal dimensional chambers in the Mori chamber decomposition of the moving cone, by Proposition 2.8, after possibly shrinking $S$, if $\left\{\mathcal{X}^{j}\right\}$ are the birational models of $\mathcal{X}$ corresponding to the maximal dimensional chambers in the Mori chamber decomposition of $\operatorname{Mov}(\mathcal{X} / S)$, then the $\mathcal{X}_{s_{i}}^{j}=\mathcal{X}^{j} \times_{S} s_{i}$ are the SQMs of the $\mathcal{X}_{s_{i}}$.

We now consider the general case. Let $\mathcal{X}_{s_{i}}^{\prime} \rightarrow \mathcal{X}_{s_{i}}$ be a small birational map and $\mathcal{B}_{s_{i}}^{\prime}$ the proper transform of $\mathcal{B}_{s_{i}}$. Since $\left(\mathcal{X}_{s_{i}}, \mathcal{B}_{s_{i}}\right)$ is klt and $\mathcal{B}_{s_{i}}$ is big, it is easy to see that $\left(\mathcal{X}_{s_{i}}^{\prime}, \mathcal{B}_{s_{i}}^{\prime}\right)$ is klt and $\mathcal{B}_{s_{i}}^{\prime}$ is big. Thus there is a $\mathbb{Q}$-factorialization $\phi: \mathcal{X}_{s_{i}}^{\prime \prime} \rightarrow \mathcal{X}_{s_{i}}^{\prime}($ see $[$ BCHM10, 1.4.3]). By what we have 
shown above, we may pick $\mathcal{X}^{p}$ which is a SQM of $\mathcal{X}$, such that

$$
\mathcal{X}_{s_{i}}^{p} \cong \mathcal{X}_{s_{i}}^{\prime \prime} .
$$

The pull back of $\operatorname{Nef}\left(\mathcal{X}_{s_{i}}^{\prime}\right)$ corresponds to a face $\Sigma$ of $\operatorname{Nef}\left(\mathcal{X}_{s_{i}}^{\prime \prime}\right)$ which in turn corresponds to a face $\Sigma$ of $\operatorname{Nef}\left(\mathcal{X}^{p} / S\right)$.

Pick a line bundle $L \in \operatorname{Nef}\left(\mathcal{X}^{p} / S\right)$ in the open chamber $\Sigma^{0}$. By (2.7), $L$ is relatively big over $S$ and so it induces a morphism $\mathcal{X}^{p} \rightarrow\left(\mathcal{X}^{p}\right)^{\prime}$ over $S$, where $\mathcal{X}_{s_{i}}^{\prime} \cong\left(\mathcal{X}^{p}\right)^{\prime} \times_{S} s_{i}$. Since $\operatorname{Nef}\left(\mathcal{X}^{p} / S\right)$ is a polyhedral cone, we conclude that there are only finitely many possible birational models $\left(\mathcal{X}^{p}\right)^{\prime}$ with a morphism $\mathcal{X}^{p} \rightarrow\left(\mathcal{X}^{p}\right)^{\prime}$. The proposition now follows by letting $\mathcal{Y} / T$ be the disjoint union of the $\left(\mathcal{X}^{p}\right)^{\prime} / S$.

\section{The main result}

Fix $I \subset[0,1]$ a DCC set and $n \in \mathbb{N}$. Let $(X, B)$ be a klt pair such that the restriction of $B$ to the generic fiber of the Iitaka fibration is $\operatorname{big}, \operatorname{dim} X=n$ and $B \in I$. The goal is to find an effective integer $m=m(n, I)$ such that $\left|m\left(K_{X}+B\right)\right|$ induces the Iitaka fibration. If $\kappa\left(K_{X}+B\right)=0$ this simply means $h^{0}\left(\mathcal{O}_{X}\left(m\left(K_{X}+B\right)\right)\right) \neq 0$. Recall the following result from [HMX14, $1.7]$.

Proposition 3.1. Fix $I \subset[0,1] \cap \mathbb{Q}$ a DCC set and $n \in \mathbb{N}$. Let $\mathcal{G}(n, I)$ be the collection of all $n$-dimensional projective klt pairs $(X, B)$ with $K_{X}+B \equiv$ $0,-K_{X}$ is ample and the coefficients of $B$ are contained in $I$. Then $\mathcal{G}(n, I)$ is bounded.

Proof. [HMX14, 1.7] proves the statement assuming that there is an $\epsilon>0$ such that for any $(X, B) \in \mathcal{G}(n, I)$ the total discrepancy of $(X, B)$ is greater than $-1+\epsilon$. Thus it suffices to prove that such an $\epsilon>0$ exists.

Suppose that $\left(X_{i}, B_{i}\right) \in \mathcal{G}(n, I)$ is a sequence of pairs with $\lim \epsilon_{i}=0$ where $-1+\epsilon_{i}>0$ is the total discrepancy of $\left(X_{i}, B_{i}\right)$. Passing to a subsequence, we may assume that $\epsilon_{i}$ is decreasing and $\epsilon_{i} \leq 1$. Let $\left(X_{i}^{\prime}, B_{i}^{\prime}\right) \rightarrow$ $\left(X_{i}, B_{i}\right)$ be the pairs obtained by extracting a divisor of discrepancy $-1+\epsilon_{i}$, then the coefficients of $B_{i}^{\prime}$ belong to the DCC set $J=I \cup\left\{1-\epsilon_{i} \mid i \in \mathbb{N}\right\}$. Hence the pairs $\left(X_{i}^{\prime}, B_{i}^{\prime}\right)$ violate [HMX14, 1.5], a contradiction.

Proof of Theorem 1.3. Let $(X, B) \in \mathcal{D}(n, I)$. By [BCHM10], running the $\left(K_{X}+B\right)$-mmp with scaling of an ample divisor, we may assume that $(X, B)$ is a good minimal model, i.e. that $K_{X}+B \sim_{\mathbb{Q}} 0$ and hence $(X, B) \in \mathcal{C}(n, I)$. Therefore, it suffices to show that $\mathcal{C}(n, I)$ is bounded. 
Let $(X, B) \in \mathcal{C}(n, I)$. The same argument as in the proof of (3.1) implies that there is an $\epsilon>0$ which only depends on $n$ and $I$ such that $(X, B)$ is $\epsilon$ klt. Let $\nu: X \rightarrow X^{\prime}$ be a minimal model for $K_{X}+(1+a) B$ and $h: X^{\prime} \rightarrow Z$ the corresponding log canonical model where $0<a \ll 1$ is chosen so that $(X,(1+a) B)$ is klt. By boundedness of log canonical models [BCHM10, 1.1.5], we may assume that $Z$ is independent of $a$. In particular, $h_{*} B^{\prime}$ is $\mathbb{Q}$-Cartier, where $B^{\prime}=\nu_{*} B$. Since

$$
K_{Z}+h_{*}\left(B^{\prime}\right) \sim(h \circ \nu)_{*}\left(K_{X}+B\right) \sim_{\mathbb{Q}} 0
$$

it follows that $K_{Z}+(1+a) h_{*}\left(B^{\prime}\right) \sim_{\mathbb{Q}} a h_{*}\left(B^{\prime}\right)$ is ample. Hence $-K_{Z} \sim_{\mathbb{Q}}$ $h_{*} B^{\prime}$ is ample. By (3.1), the set $\mathcal{C}^{\prime}$ of all such pairs $\left(Z, h_{*} B^{\prime}\right)$ is bounded. Note that $\left(Z, h_{*} B^{\prime}\right)$ is $\epsilon$-klt (see the proof of (3.1)) for some fixed $\epsilon>0$ which only depends on $n$ and $I$, so we can apply (2.5) and denote by $\mathcal{C}^{\prime \prime}$ the bounded family constructed there.

For any $\left(Z, h_{*} B^{\prime}\right) \in \mathcal{C}^{\prime}$, consider a common resolution $p: Y \rightarrow X$ and $q: Y \rightarrow Z$, since

$$
p^{*}\left(K_{X}+B\right)=q^{*}\left(K_{Z}+h_{*} B^{\prime}\right),
$$

it follows that the discrepancy of any $q \circ p^{-1}$-exceptional divisor $E \subset X$ is $\leq 0$ as

$$
a\left(E, Z, h_{*} B^{\prime}\right)=a(E, X, B) \leq 0 .
$$

Hence, there exists a proper birational morphism $g: X^{\prime \prime} \rightarrow Z$ such that the induced rational map $\psi: X \rightarrow X^{\prime \prime}$ is an isomorphism in codimension 1 and $\left(X^{\prime \prime}, B^{\prime \prime}:=\psi_{*} B\right)$ belongs to $\mathcal{C}^{\prime \prime}$.

Finally, note that as $B$ is big, so is $B^{\prime \prime}$ and $\left(X^{\prime \prime}, B^{\prime \prime}\right)$ is $\epsilon$-klt, $\mathbb{Q}$-factorial with $K_{X^{\prime \prime}}+B^{\prime \prime} \sim_{\mathbb{Q}} 0$. By (2.9) it follows that $\mathcal{C}$ is bounded.

Proof of (1.5). By [Kol93], we know that there exists a uniform $N=N(m, n)$ such that $\left|-N K_{X}\right|$ is base point free. Let $D$ be a general divisor in $\mid-$ $N K_{X} \mid$, then the pair $\left(X, \frac{1}{N} D\right)$ satisfies the assumption of Theorem 1.3 and the assertion follows.

Proof of (1.4). By [Lai11, 4.4], we may replace $(X, B)$ by a good minimal model and hence we may assume that $K_{X}+B$ is semiample. We note that in [Lai11, 4.4], it is assumed that $X$ is terminal. However, the same argument can be applied if we only assume the pair $(X, B)$ to be klt, since in the argument the only used facts are that we can run MMP of $(X, B)$ with a scaling of an ample divisor, and the pluricanonical ring of $(X, B)$ is finitely generated. Both of these facts follow from [BCHM10]. Alternatively this follows from [HX13, 2.10, 2.12]. 
Let $Z=\operatorname{Proj}\left(R\left(K_{X}+B\right)\right)$ and $f: X \rightarrow Z$ the induced morphism which coincides with the Iitaka fibration. We can write

$$
K_{X}+B \sim_{\mathbb{Q}} f^{*}\left(K_{Z}+B_{Z}+M_{Z}\right)
$$

where $B_{Z}$ is the boundary part and $M_{Z}$ is the moduli part.

Let $\nu: Z^{\prime} \rightarrow Z$ be a resolution of $Z$ and $X^{\prime \prime}$ be a resolution of the main component of $X \times_{Z} Z^{\prime}, \mu: X^{\prime \prime} \rightarrow X$ the induced morphism. Write

$$
K_{X^{\prime \prime}}+B^{\prime \prime}=\mu^{*}\left(K_{X}+B\right)+\sum a_{i} E_{i}
$$

such that each $E_{i}$ is exceptional, and the coefficients of $B^{\prime \prime}$ are contained in the DCC set $I^{\prime}=I \cup\left\{\frac{n-1}{n} \mid n \in \mathbb{N}\right\}$ and $a_{i}>0$ for all exceptional divisors $E_{i} . B^{\prime \prime}$ is big over $Z^{\prime}$ and so we can run a relative MMP of $\left(X^{\prime \prime}, B^{\prime \prime}\right)$ over $Z^{\prime}$. Let $f^{\prime}:\left(X^{\prime}, B^{\prime}\right) \rightarrow Z^{\prime}$ be the corresponding minimal model over $Z^{\prime}$.

We may apply [Kaw98, Theorem 2] and write

$$
K_{X^{\prime}}+B^{\prime} \sim_{\mathbb{Q}} f^{\prime *}\left(K_{Z^{\prime}}+B_{Z^{\prime}}+M_{Z^{\prime}}\right)
$$

where $B_{Z^{\prime}}$ is the boundary part (in [Kaw98, Theorem 2], it is denoted by $\Delta$ ) and $M_{Z^{\prime}}$ is the moduli part. In particular, choosing $Z^{\prime}$ and $X^{\prime \prime}$ appropriately, we may assume that $M_{Z^{\prime}}$ is nef.

Claim 3.2. There exists an integer $r>0$ and a DCC set $\Lambda$ such that

1) $\left(Z^{\prime}, B_{Z^{\prime}}\right)$ is klt,

2) the coefficients of $B_{Z}$, belong to $\Lambda$,

3) $r M_{Z^{\prime}}$ is a nef Cartier divisor, and

4) $K_{Z^{\prime}}+B_{Z^{\prime}}+M_{Z^{\prime}}$ is big.

Since $f^{\prime}$ is birational to the Iitaka fibration of $(X, B)$ and

$$
K_{X^{\prime}}+B^{\prime} \sim_{\mathbb{Q}} f^{\prime *}\left(K_{Z^{\prime}}+B_{Z^{\prime}}+M_{Z^{\prime}}\right)
$$

it follows that (4) holds.

To check that (3) holds, we follow [FM00, Theorem 3.1] which is known to apply to the log setting, see for example [Tod10, $\S 3]$. Let $F$ be the general fiber of $f$ and write $\left.\left(K_{X}+B\right)\right|_{F}=\left(K_{F}+B_{F}\right)$, then $K_{F}+B_{F} \sim_{\mathbb{Q}} 0$ and $B_{F}$ is big. So by $(1.3),\left(F, B_{F}\right)$ belongs to the bounded family $\mathcal{C}(\rho, I)$ where $\rho=\operatorname{dim} X-\operatorname{dim} Z$. In particular, there exists an integer $b>0$ which only depends on $\Lambda$ and $\rho$ such that $b\left(K_{F}+B_{F}\right) \sim 0$. 
We let $\eta: F^{\prime \prime} \rightarrow F$ be a $\log$ resolution of $\left(F, B_{F}\right)$. We write

$$
K_{F^{\prime \prime}}+B_{F^{\prime \prime}}=\eta^{*}\left(K_{F}+B_{F}\right)
$$

then

$$
D_{F^{\prime \prime}}:=b\left\{B_{F^{\prime \prime}}\right\} \sim_{\mathbb{Q}} b\left(-K_{F^{\prime \prime}}-\left\lfloor B_{F^{\prime \prime}}\right\rfloor\right) .
$$

In particular, $D_{F^{\prime \prime}}$ is an effective divisor.

We denote by $\phi: F^{\prime \prime \prime} \rightarrow F^{\prime \prime}$ the corresponding degree $b$ cyclic covering branched over $D_{F^{\prime \prime}}$ and $\psi: F^{\prime} \rightarrow F^{\prime \prime \prime}$ a resolution. Since $\left(F, B_{F}\right) \in \mathcal{C}(\rho, I)$ and $\mathcal{C}(\rho, I)$ is bounded, it is easy to see that we can assume that $\left(F^{\prime}, B_{F^{\prime}}\right) \in$ $\mathcal{C}^{\prime}$ where $K_{F^{\prime}}+B_{F^{\prime}}=(\eta \circ \phi \circ \psi)^{*}\left(K_{F}+B_{F}\right)$ and $\mathcal{C}^{\prime}$ is bounded. In particular, it follows that the middle Betti number $B_{\rho}\left(F^{\prime}\right)$ is bounded by a constant $l$.

Lemma 3.3. Let $\varphi$ be the Euler function, then there exists a positive integer $r$ such that $r M_{Z^{\prime}}$ is Cartier and $\varphi(r) \leq l$.

Proof. When $F$ is smooth and $B_{F}=0$, this is [FM00, Theorem 3.1]. The general case is well known and similar to [FM00]. For the reader's convenience, we include a short sketch.

Following [FM00, 3.6], fix any divisor $P$ on $Z^{\prime}$, let $(Q \in W) \rightarrow\left(P \in Z^{\prime}\right)$ be a base change, such that $X^{\prime} \times Z_{Z^{\prime}} W$ admits a semistable model over a neighborhood of the generic point of $Q$. Then $r M_{Z^{\prime}}$ is integral at $P$ if the local monodromy action on $H^{0}\left(F^{\prime}, K_{F^{\prime}}\right.$ ) (where $F^{\prime}$ is considered as a fiber over $W$ ) factors through

$$
\mu_{r}: \mathbb{Z} / r \mathbb{Z} \rightarrow \mathbb{C}^{*}
$$

On the other hand, there is a $\mu_{r}$-equivariant injection

$$
H^{0}\left(F^{\prime}, K_{F^{\prime}}\right) \subset H^{\rho}\left(F^{\prime}, \mathbb{Z}\right) \otimes \mathbb{C}
$$

where $H^{\rho}\left(F^{\prime}, \mathbb{Z}\right)$ is the middle Betti cohomology of $F^{\prime}$. The eigenvalues of the corresponding action on $H^{\rho}\left(F^{\prime}, \mathbb{Z}\right) \otimes \mathbb{C}$ are always algebraic integers, thus we conclude $\varphi(r) \leq \operatorname{dim}_{\mathbb{C}}\left(H^{\rho}\left(F^{\prime}, \mathbb{Z}\right) \otimes \mathbb{C}\right)=l($ cf. [FM00, 3.7, 3.8]).

To check that (2) holds, recall that for a codimension 1 point $P$ on $Z$, its coefficient in $B_{Z^{\prime}}$ is equal to

$$
1-\operatorname{lct}_{f^{\prime-1}(P)}\left(X^{\prime}, B^{\prime}, f^{\prime *}(P)\right)
$$


where the log canonical threshold is computed locally around $f^{\prime-1}(P)$ (cf. [Kaw98], [Amb99, 3.1.4]). Therefore, by [HMX14, Theorem 1.1], it is contained in a DCC set.

By [BZ14, 1.2], it follows that there is an integer $m$ depending only on $\Lambda$, $r$ and $d=\operatorname{dim} Z$ such that $\left|m\left(K_{Z^{\prime}}+B_{Z^{\prime}}+M_{Z^{\prime}}\right)\right|$ defines a birational map. Since

$$
H^{0}\left(X^{\prime}, m b\left(K_{X^{\prime}}+B^{\prime}\right)\right) \cong H^{0}\left(Z^{\prime}, m b\left(K_{Z^{\prime}}+B_{Z^{\prime}}+M_{Z^{\prime}}\right)\right),
$$

it follows that $\left|m b\left(K_{X^{\prime}}+B^{\prime}\right)\right|$ defines the Iitaka fibration. This concludes the proof of (1.4).

\section{References}

[Ale94] Valery Alexeev, Boundedness and $K^{2}$ for log surfaces. Internat. J. Math., 5 (1994), no. 6, 779-810.

[Amb99] Florin Ambro, The Adjunction Conjecture and its applications. arXiv: 9903060 (1999).

[BCHM10] Caucher Birkar, Paolo Cascini, Christopher D. Hacon and James McKernan, Existence of minimal models for varieties of log general type. J. Amer. Math. Soc., 23 (2010), no. 2, 405-468.

[BL15] Lev Borisov and Zhani Li, On complete intersection with trivial canonical class. Adv. Math., 268 (2015), 339-349.

[BZ14] Caucher Birkar and De-Qi Zhang, Effectivity of Iitaka fibrations and pluricanonical systems of polarized pairs. arXiv:1410.0938 (2014).

[CC10] Jungkai Chen and Meng Chen, Explicit birational geometry of threefolds of general type, I. Ann. Sci. École Norm. Supér. (4), 43 (2010), no. 3, 365-394.

[CL12] Paolo Cascini and Vladimir Lazić, New outlook on the minimal model program, I. Duke Math. J., 161 (2012), no. 12, 2415-2467.

[dFH11] Tommaso de Fernex and Christopher D. Hacon, Deformations of canonical pairs and Fano varieties. J. Reine Angew. Math., 651 (2011), 97-126.

[FM00] Osamu Fujino and Shigefumi Mori, A canonical bundle formula. J. Differential Geometry, 56 (2000), 167-188. 
[HK00] Yi Hu and Sean Keel, Mori dream spaces and GIT. Michigan Math. J., 48 (2000), 331-348.

[HM06] Christopher D. Hacon and James McKernan, Boundedness of pluricanonical maps of varieties of general type. Invent. Math., 166 (2006), no. 1, 1-25.

[HX13] Christopher D. Hacon and Chenyang Xu, Existence of log canonical closures. Invent. Math. (2013), 161-195.

[HMX13] Christopher D. Hacon, James McKernan and Chenyang Xu, On the birational automorphisms of varieties of general type. Ann. of Math. (2), 177 (2013), no. 3, 1077-1111.

[HMX14] Christopher D. Hacon, James McKernan and Chenyang Xu, ACC for log canonical thresholds. Ann. of Math. (2), 180 (2014), no. 2, 523-571.

[Jia13] Xiaodong Jiang, On the pluricanonical maps of varieties of intermediate Kodaira dimension. Math. Ann., 356 (2013), no. 3, 9791004 .

[Kaw86] Yujiro Kawamata, On the plurigenera of minimal algebraic 3folds with $K_{X} \equiv 0$. Math. Ann., 275 (1986), no. 4, 539-546.

[Kaw98] Yujiro Kawamata, Subadjunction of log canonical divisors. II. Amer. J. Math., 120 (1998), no. 5, 893-899.

[Kol93] János Kollár, Effective base point freeness. Math. Ann., 296 (1993), no. 4, 595-605.

[Kol94] János Kollár, Log surfaces of general type; some conjectures. Classification of algebraic varieties (L'Aquila, 1992), 1994, pp. 261-275.

[KM92] János Kollár and Shigefumi Mori, Classification of threedimensional flips. J. Amer. Math. Soc., 5 (1992), no. 3, 533-703.

[KM98] János Kollár and Shigefumi Mori, Birational geometry of algebraic varieties. Cambridge Tracts in Mathematics, vol. 134, Cambridge University Press, Cambridge, 1998. With the collaboration of C. H. Clemens and A. Corti; Translated from the 1998 Japanese original.

[Lai11] Ching-Jui Lai, Varieties fibered by good minimal models. Math. Ann., 350 (2011), no. 3, 533-547. 
[Laz04] Robert Lazarsfeld, Positivity in algebraic geometry. I. Ergebnisse der Mathematik und ihrer Grenzgebiete. 3. Folge. A Series of Modern Surveys in Mathematics, vol. 48, Springer-Verlag, Berlin, 2004.

[PS06] Yu. G. Prokhorov and V. V. Shokurov, Toward the Second Main Theorem on Complements: From Local to Global. MPI / MaxPlanck-Institut für Mathematik, Bonn (2006).

[Tak06] Shigeharu Takayama, Pluricanonical systems on algebraic varieties of general type. Invent. Math., 165 (2006), 551-587.

[Tod10] Gueorgui Tomov Todorov, Effective log Iitaka fibrations for surfaces and threefolds. Manuscripta Math., 133 (2010), no. 1-2, 183-195.

[Tsu06] Hajime Tsuji, Pluricanonical systems of projective varieties of general type. I. Osaka J. Math., 43 (2006), no. 4, 967-995.

[TX09] Gueorgui Todorov and Chenyang Xu, Effectiveness of the log Iitaka fibration for 3-folds and 4-folds. Algebra Number Theory, 3 (2009), no. 6, 697-710.

[VZ09] Eckart Viehweg and De-Qi Zhang, Effective Iitaka fibrations. J. Algebraic Geom., 18 (2009), no. 4, 711-730.

Department of Mathematics, University of Utah

155 South 1400 East, JWB 233, Salt Lake City, UT 84112, USA

E-mail address: hacon@math.utah.edu

Beijing International Center of Mathematics Research

5 Yiheyuan Road, Haidian District, Beijing 100871, China

E-mail address: cyxu@math.pku.edu.cn

Received November 23, 2014 